\title{
Objective Motion Cueing Criteria for Commercial Transport Simulators
}

Peter M. T. Zaal, San Jose State University; Jeffery A. Schroeder, Federal Aviation Administration; William W. Chung, Science Applications Internatinoal Corporation

This paper adds data to establish fidelity criteria for the simulator motion system diagnostic test now re- quired during commercial aircraft simulator approval in the United States. Nineteen airline transport pilots flew three tasks under six different motion conditions in an experiment on the NASA Vertical Motion Simula- tor. The motion conditions allowed refinement of the initial fidelity criteria developed in previous experiments. In line with these previous experiments, the motion condition significantly affected (1) false motion cue pilot ratings, and sink rate and longitudinal deviation at touchdown in the approach and landing task, (2) false motion cue pilot ratings, roll deviations, and maximum pitch rate in the stall task, and (3) false motion cue pilot ratings, heading deviation, and pedal reaction time after an engine failure in the take-off task. Combining data from three experiments, significant differences in pilot-vehicle performance were used to define objective mo- tion cueing criteria boundaries. These fidelity boundaries suggest that some hexapod simulators can possibly produce motion cues with improved fidelity in several degrees of freedom. 


\section{Objective Motion Cueing Criteria for Commercial Transport Simulators}

\section{AIAA-2018-2935}
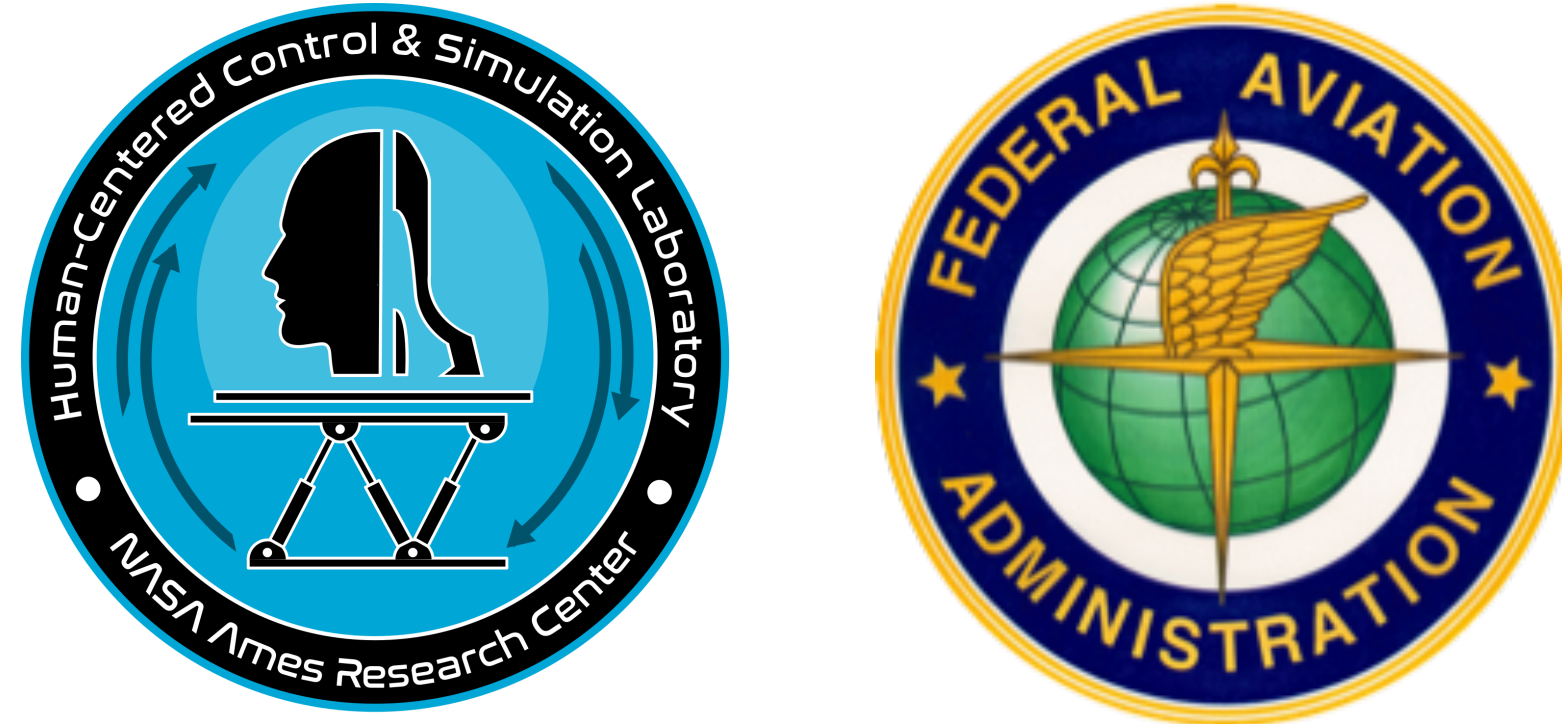

\section{Peter M. T. Zaal}

San José State University NASA Ames Research Center
Jeffery A. Schroeder

Federal Aviation Administration
William W. Chung Science Applications Int. Corp. NASA Ames Research Center 


\section{Outline}

Introduction

Tasks

Motion Conditions

Experiment design

Results

Conclusions

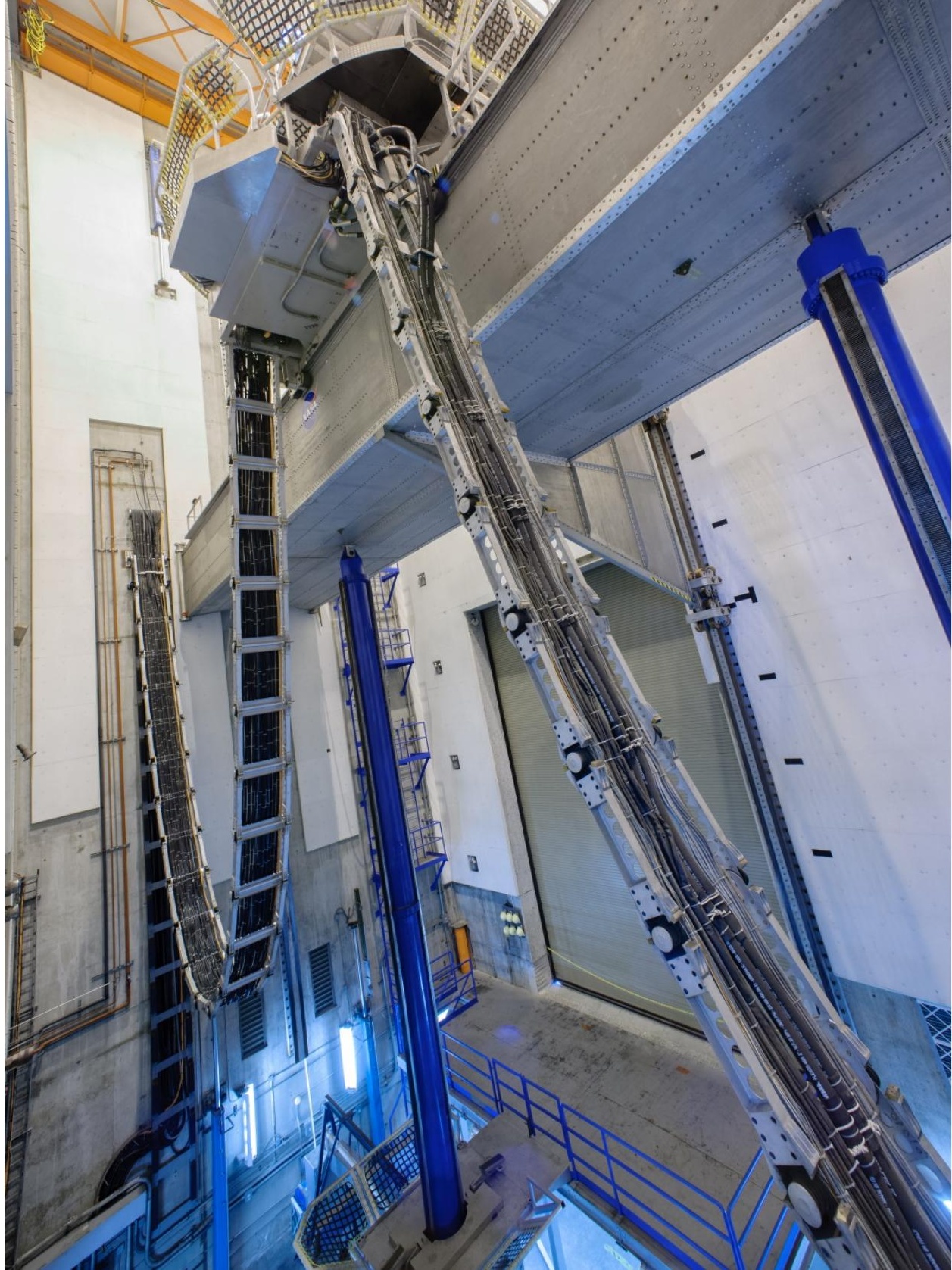




\section{Introduction}

Previously, during a simulator qualification:

- Engineers measured motion hardware

- Pilot inspectors assessed hardware + software

Now:

- FAA part 60 requires an Objective Motion Cueing Test (OMCT) for new devices

- Currently, no fidelity criteria accompany the test 


\section{Introduction}

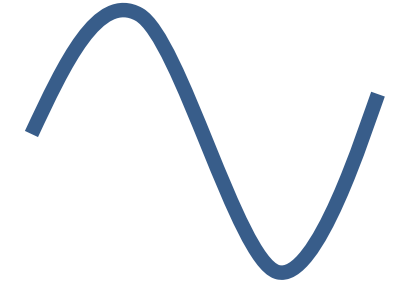

aircraft model pilot station accelerations

\section{Objective Motion Cueing Test}

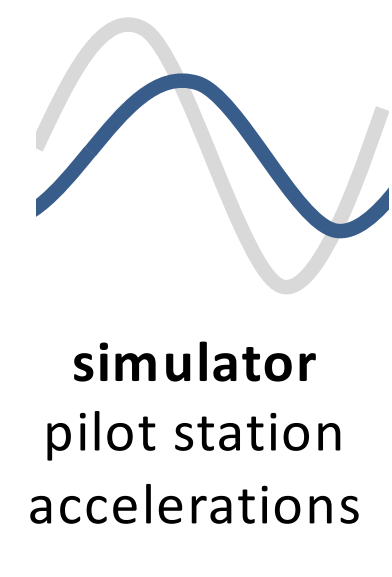

Roll magnitude

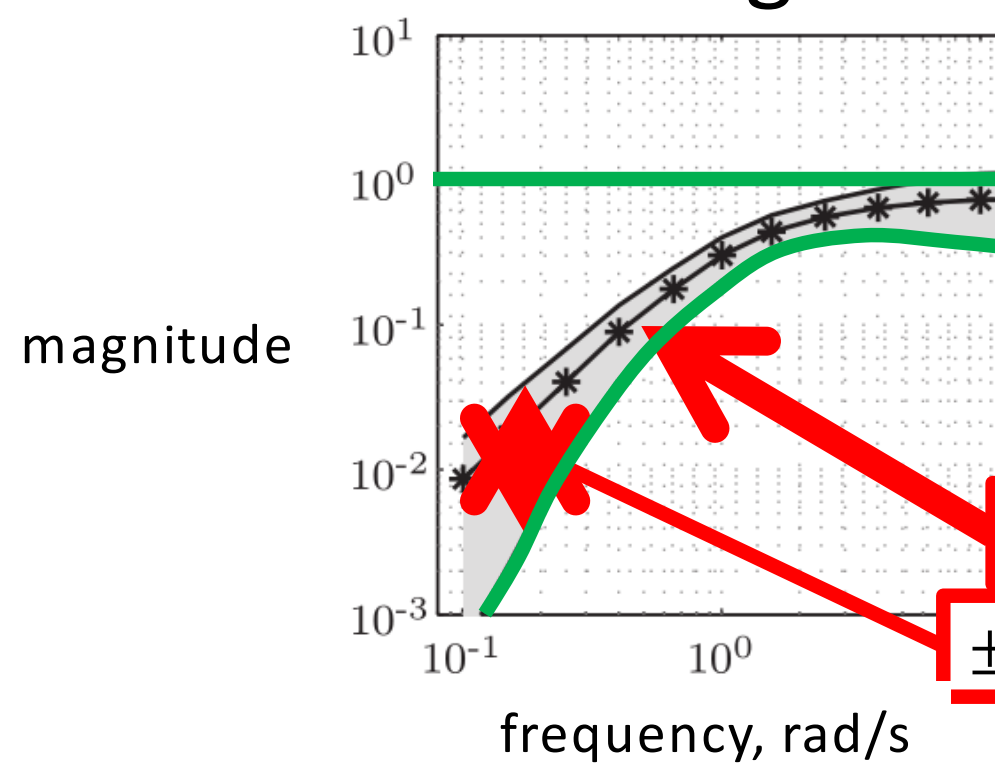

Roll phase

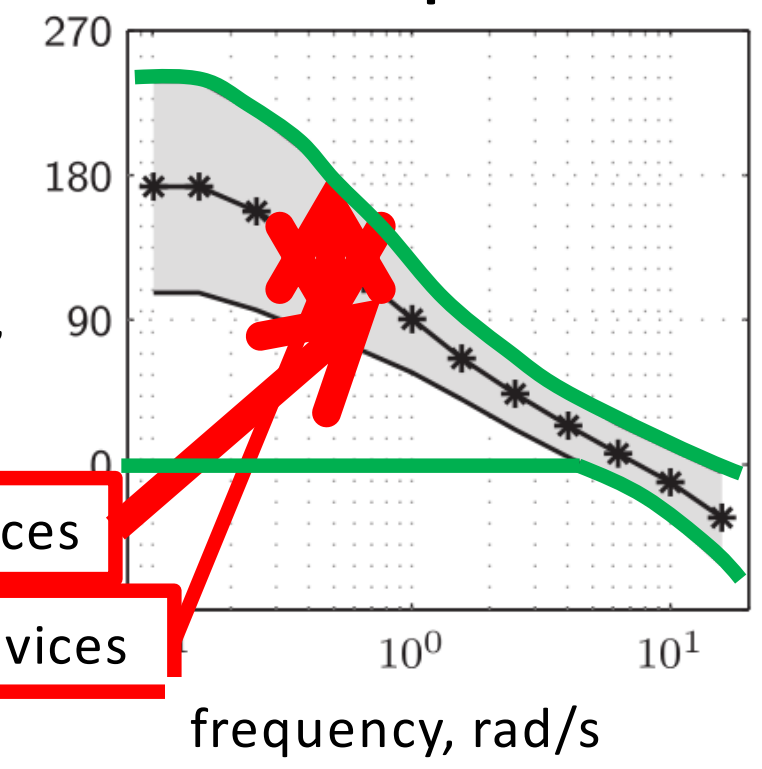




\section{Introduction}

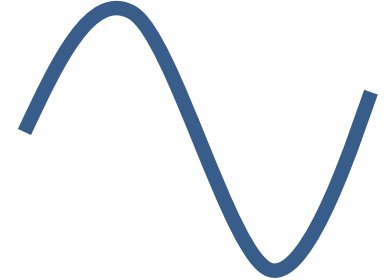

aircraft model pilot station accelerations

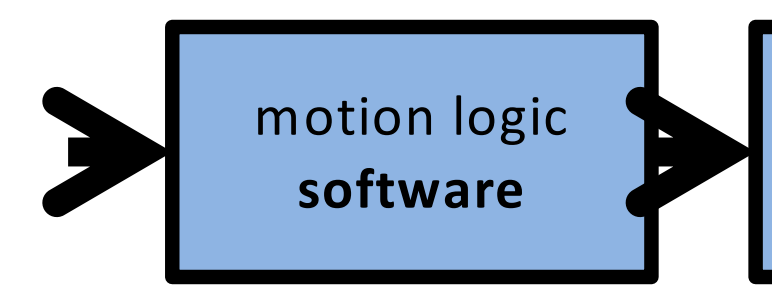

\section{Objective Motion Cueing Test}

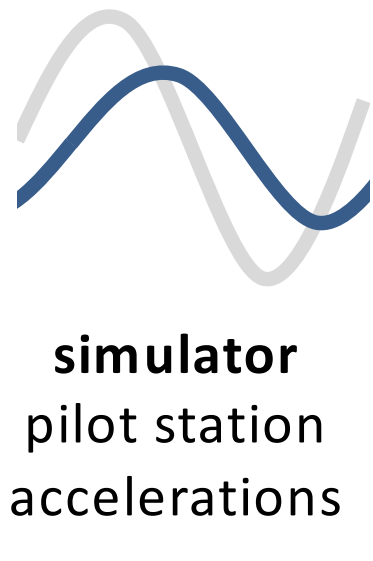

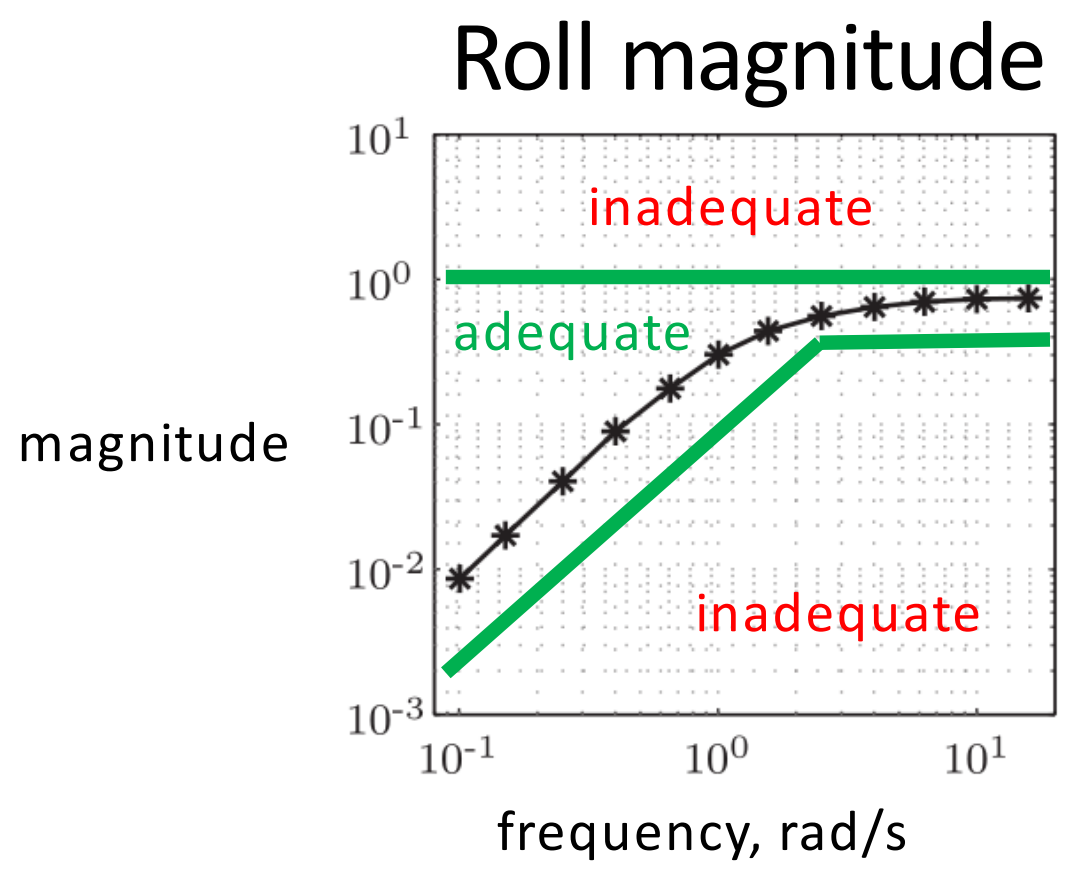

Roll phase

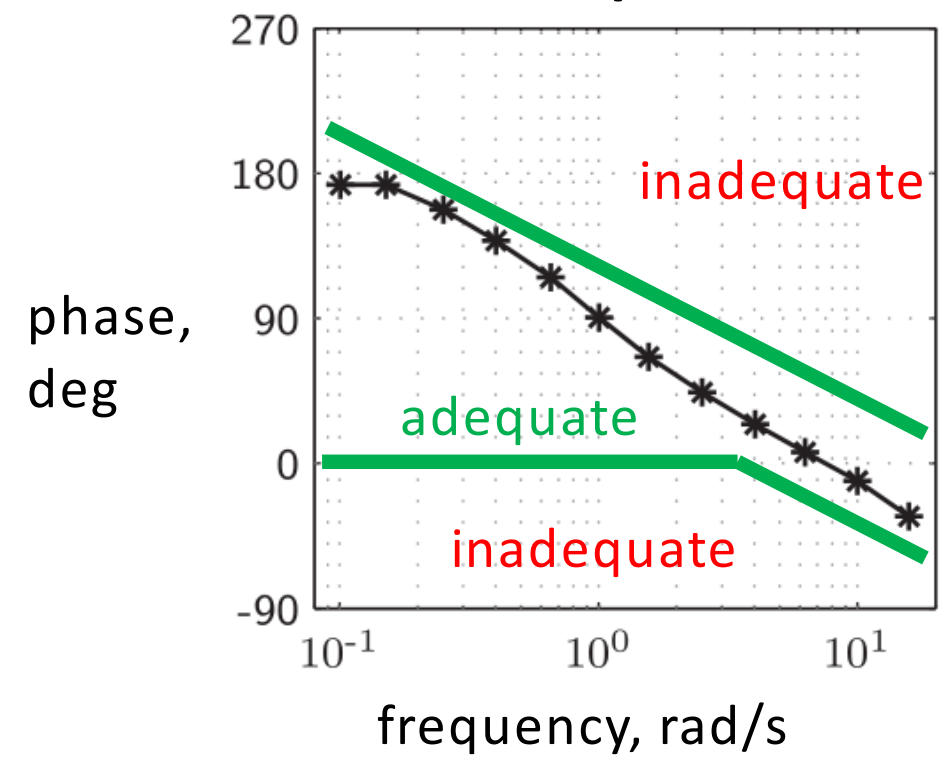




\section{Introduction}

Objective:

Develop fidelity criteria for the Objective Motion Cueing Test for Commercial Transport Simulators

What's new?

- Well-behaved transport aircraft

- Three tasks, 6 new motion configurations

- World's largest motion simulator

- Sufficiently large pilot pool 


\section{Tasks}

1. Approach and landing with sidestep

2. High-altitude stall recovery

3. Engine out on takeoff 


\section{Motion Conditions}
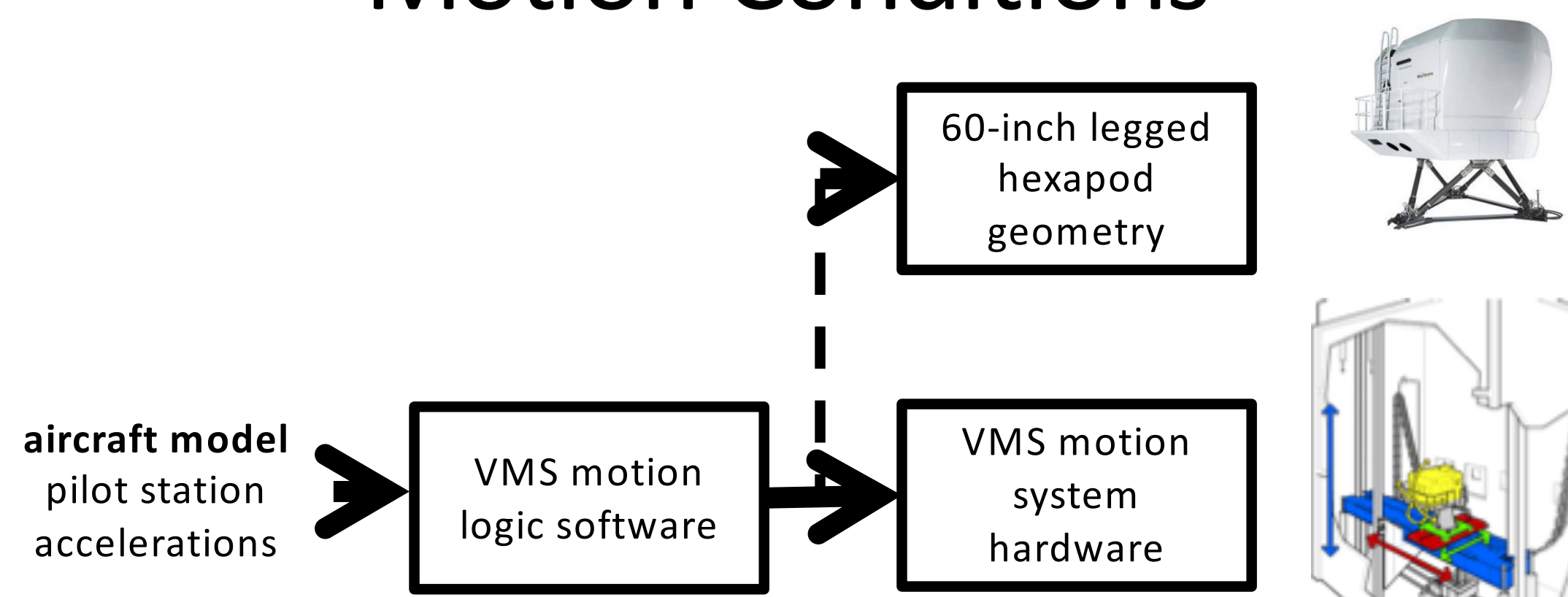

Six motion configurations per task:

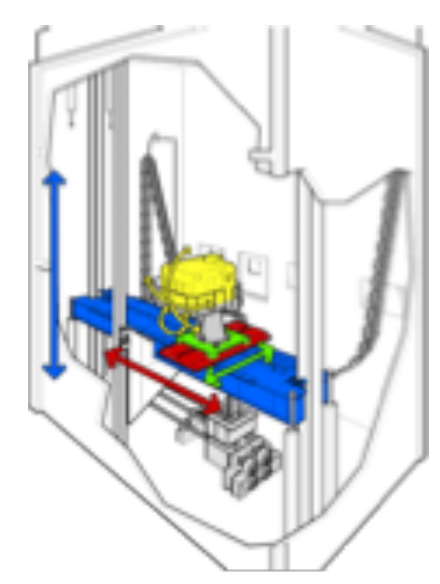

1. Gain/break-frequency tradeoff

2. Compare degrees of freedom

3. False tilt motion cues 


\section{Motion Conditions}

- Sidestep task:

1. Surge motion

2. Roll motion

3. Pitch and heave

- Stall task:

1. Roll motion

2. Roll motion

3. Pitch and heave

- Takeoff task:

1. Surge to pitch

2. Yaw and sway $\rightarrow$ Tail windshear recovery

$\rightarrow$ Perceived tilt cues in turns

$\rightarrow$ Landing flare

$\rightarrow$ Roll disturbance compensation

$\rightarrow$ Perceived tilt cues in turn

$\rightarrow$ Secondary stall occurrence

$\rightarrow$ Perceived tilt cues initial acc.

$\rightarrow$ Engine failure compensation 


\begin{abstract}
Full VMS Motion
\end{abstract}

High

Gain

Low Hexapod 


\section{Experiment Design}

- 19 airline transport pilots

- Three challenging flight tasks

- Six motion configurations per task

- Six repetitions per task and motion configuration

- B757-like aircraft model

- Cockpit:

side-by-side with

B777-like primary display

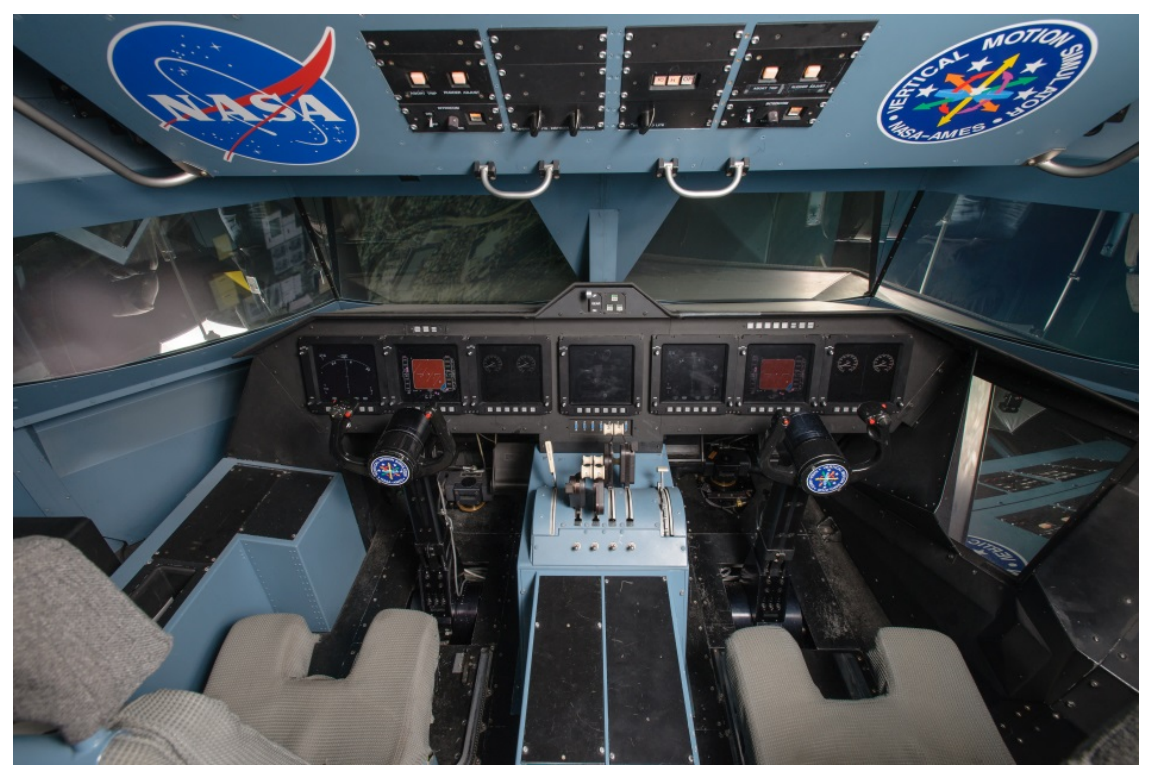




\section{Experiment Design}

- Dependent measures:

- Three subjective ratings of false tilt motion

- 12 objective task-performance measures
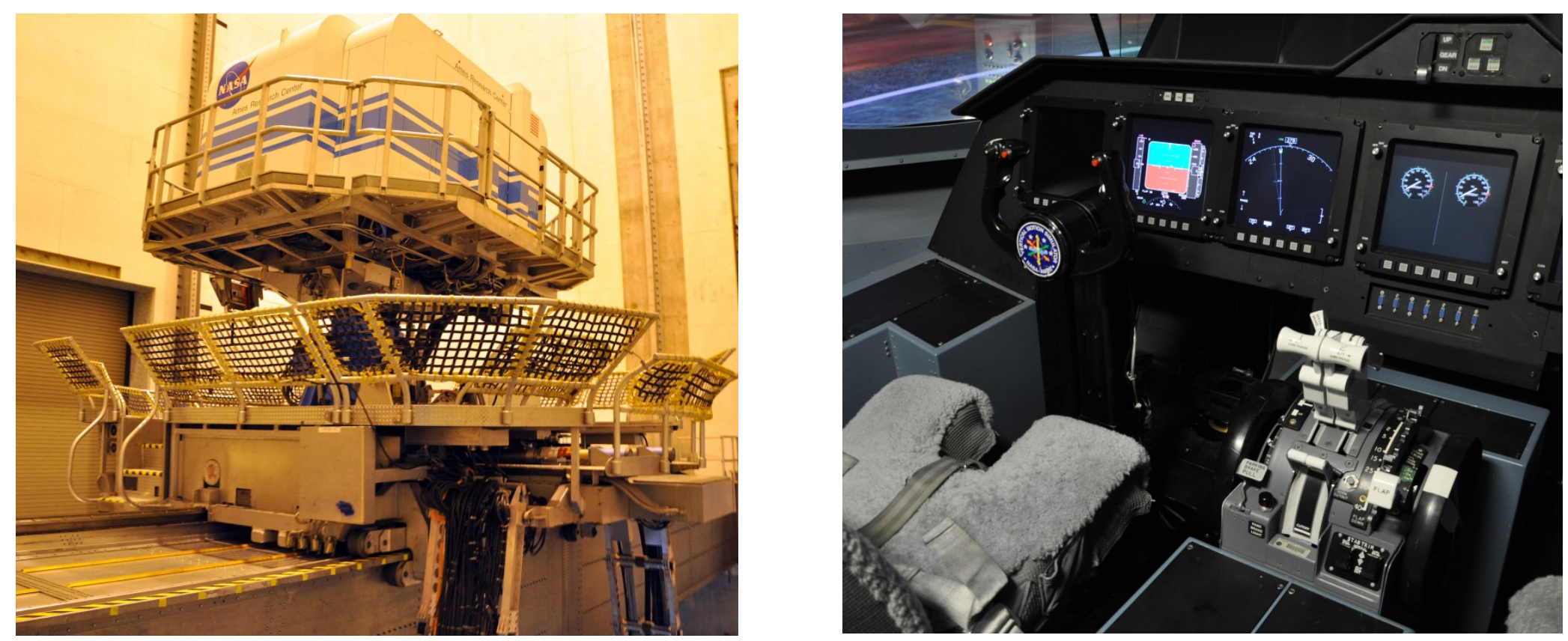


\section{Performance Results \\ Sidestep Task}

Sinkrate at Touchdown, $\mathrm{ft} / \mathrm{s}$
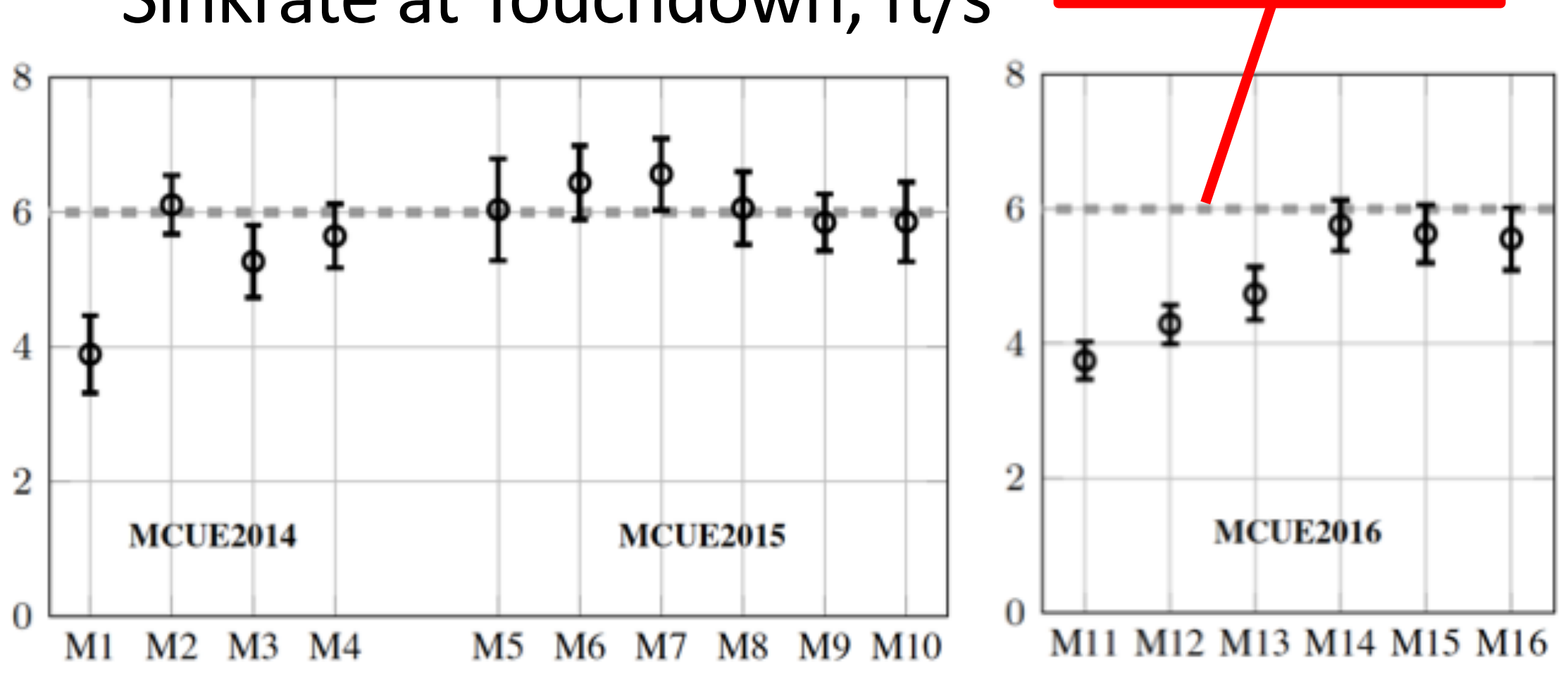

Decreasing heave gain and increasing break frequency 


\section{Performance Results High-Altitude Stall}

Roll deviation RMS, deg

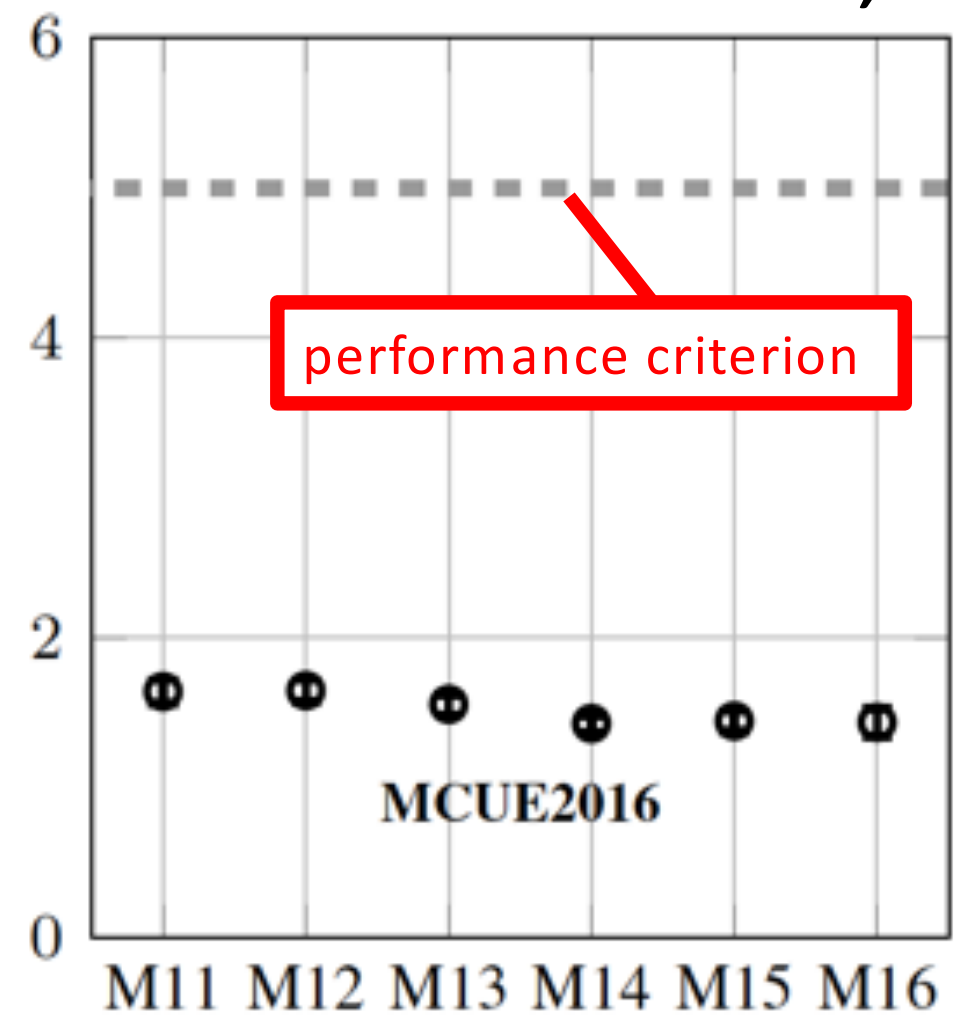

Decreasing roll break frequency
Max pitch rate, deg

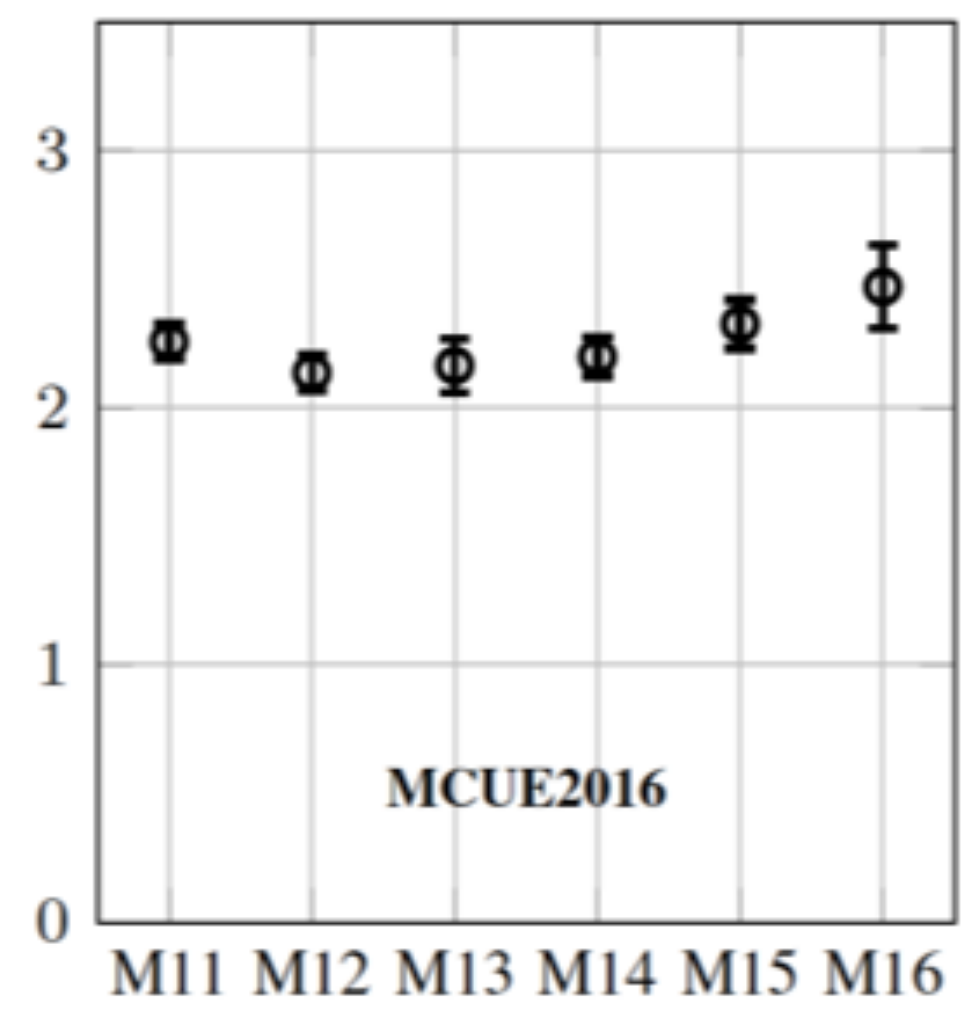

Decreasing heave fidelity and increasing pitch fidelity 


\section{Performance Results Takeoff Task}

Heading deviation RMS, deg

Pedal response time, $\mathrm{s}$
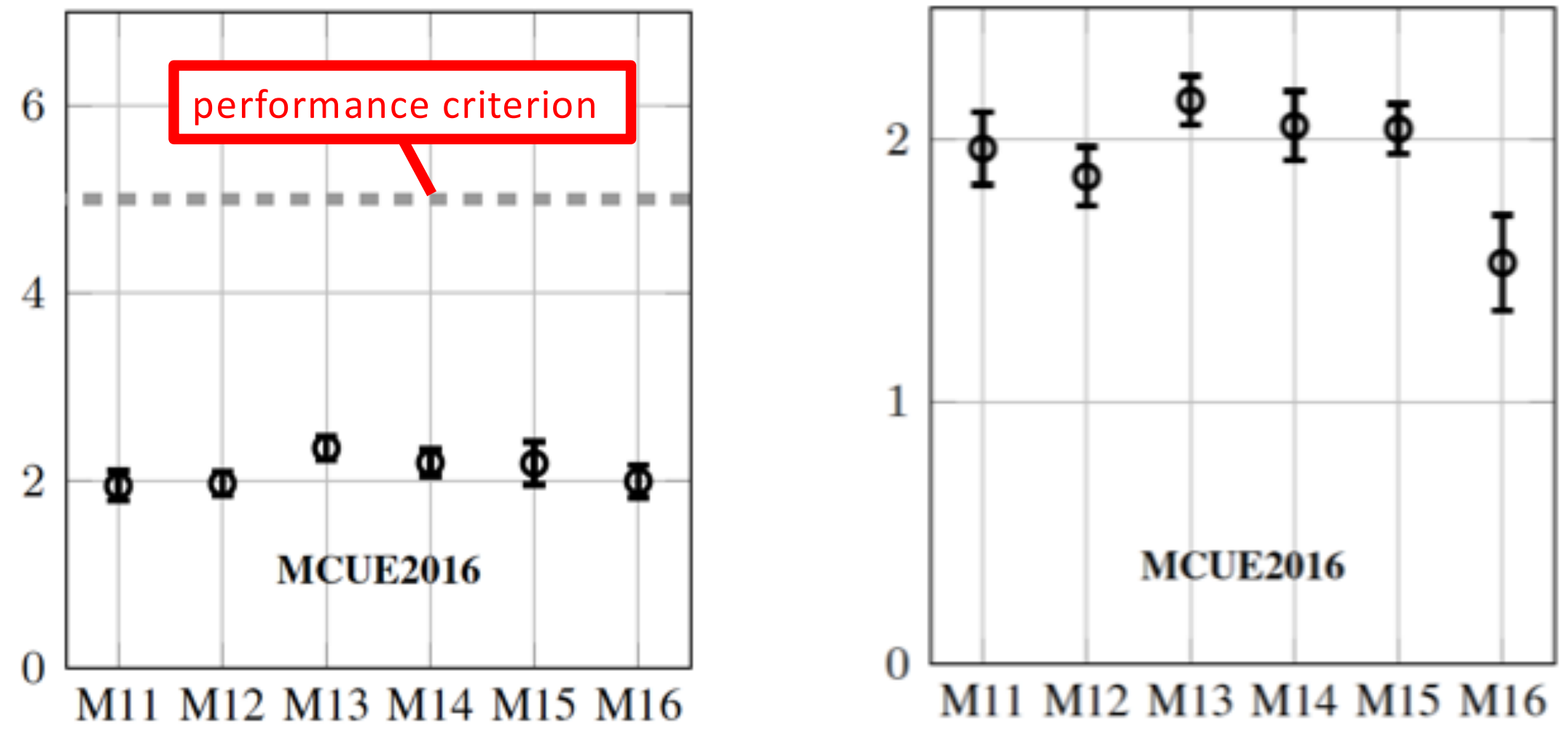

Increasing sway fidelity and decreasing yaw fidelity 


\section{Motion Rating Results Sidestep Task}
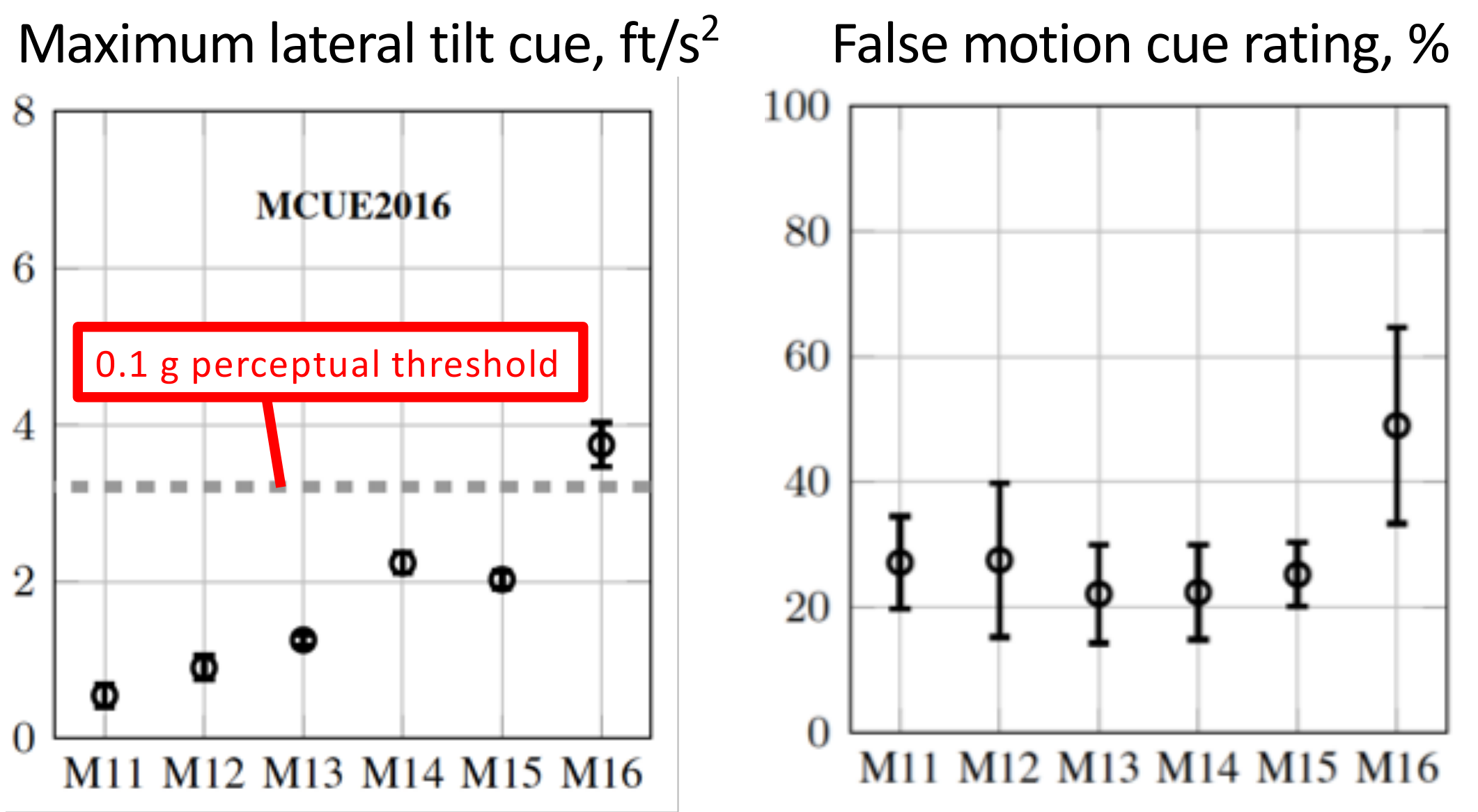

Increasing false lateral specific force cues 


\section{Motion Rating Results Takeoff Task}

Max tilt-coordination pitch rate, $\mathrm{deg} / \mathrm{s}$

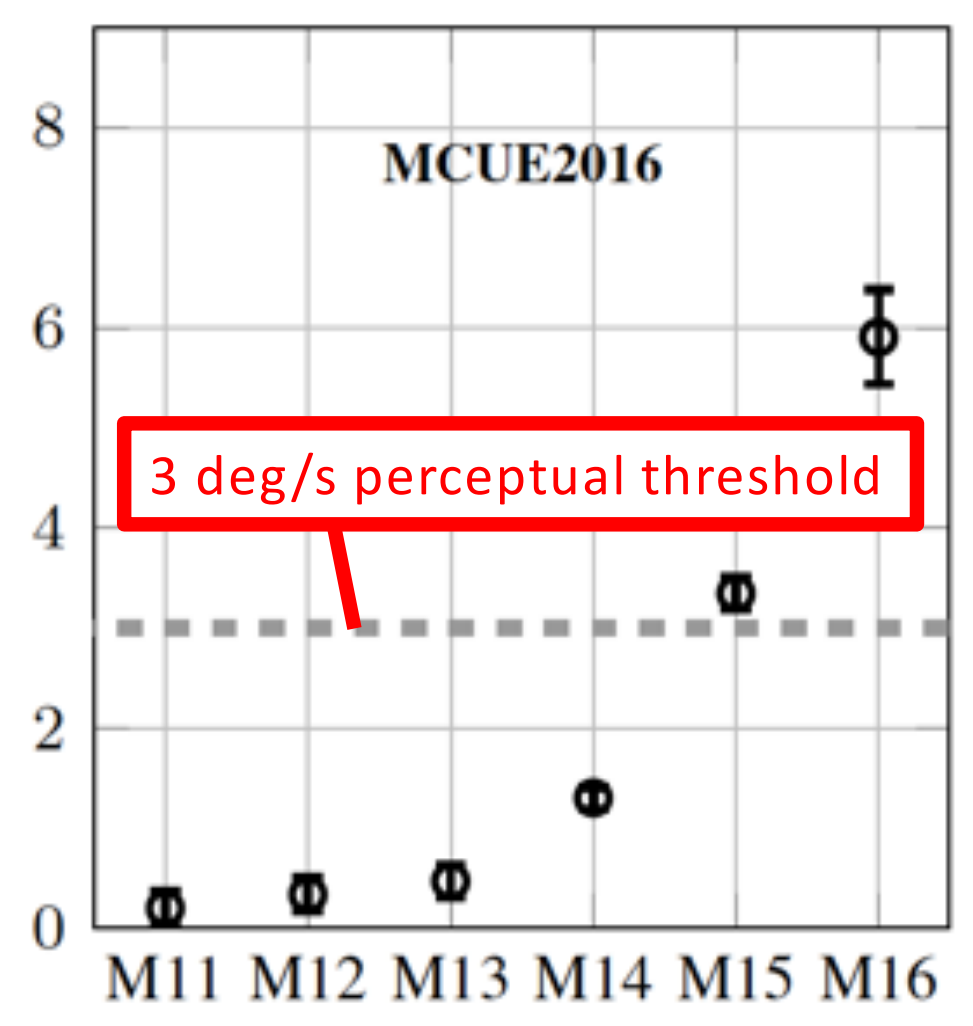

False motion cue rating, \%

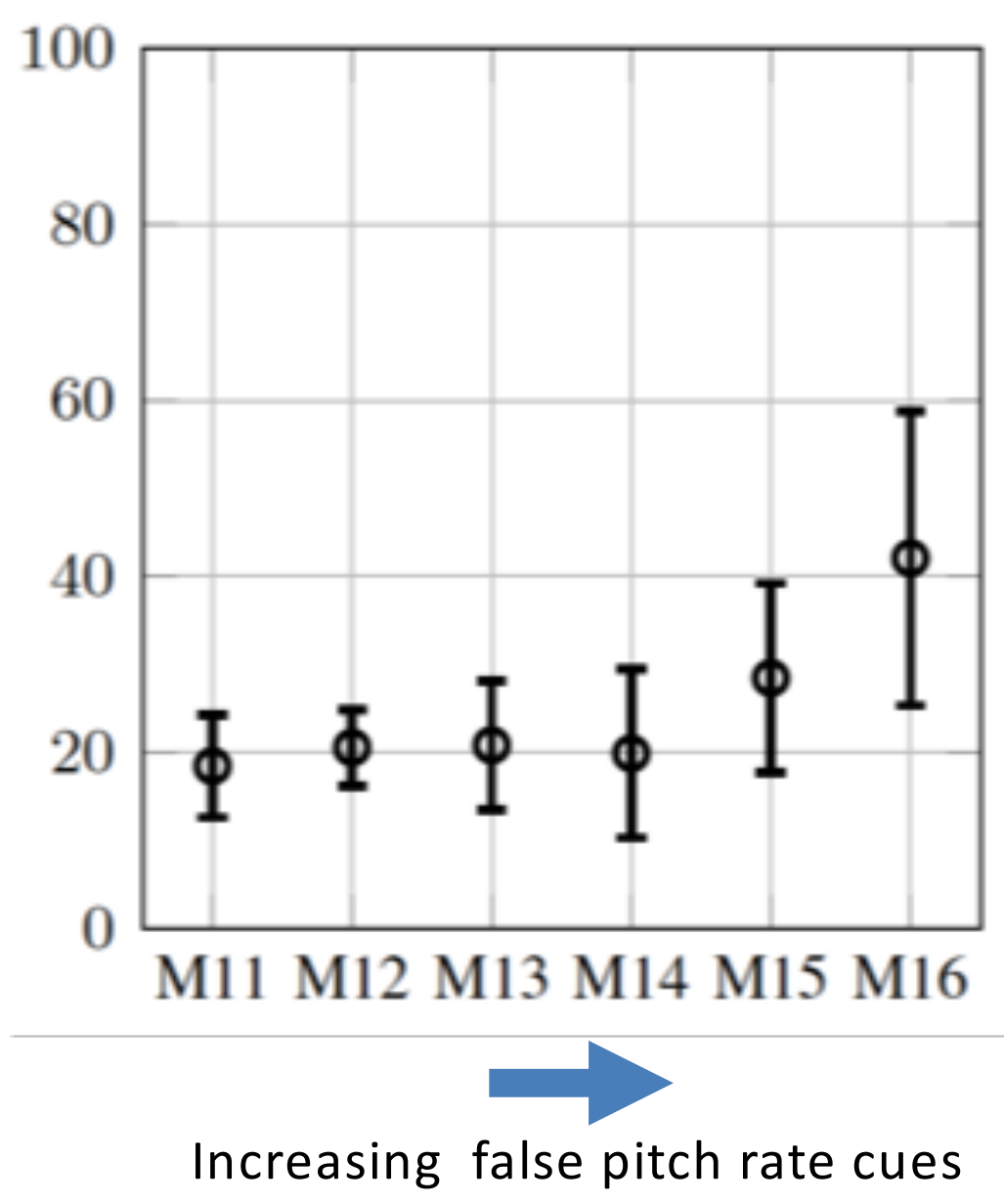




\section{Results \\ Objective Motion Cueing Criteria}

Step 1:

Touchdown sinkrate, $\mathrm{ft} / \mathrm{s}$

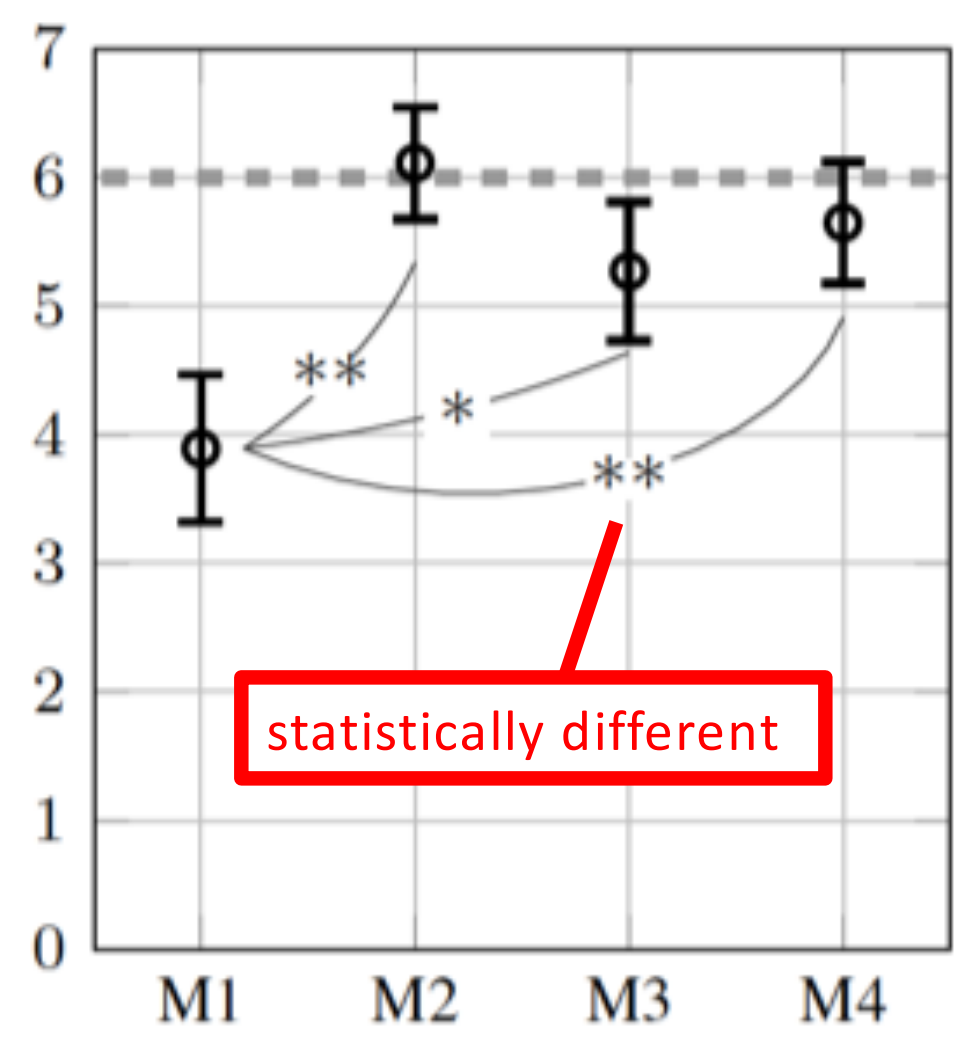

Step 2:

Heave uncertainty bounds

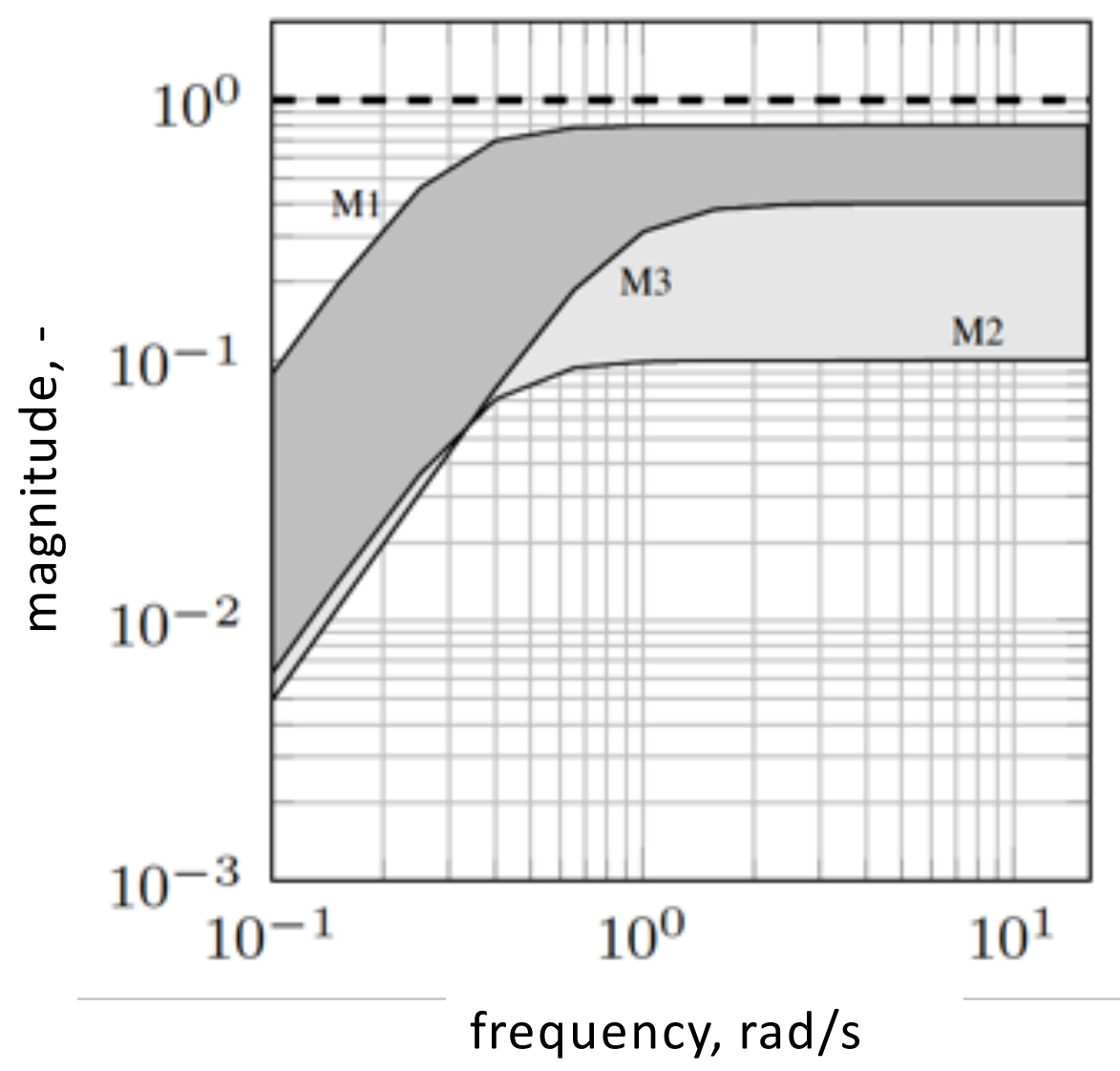




\section{Rotational Motion Cueing Bounds}

Pitch

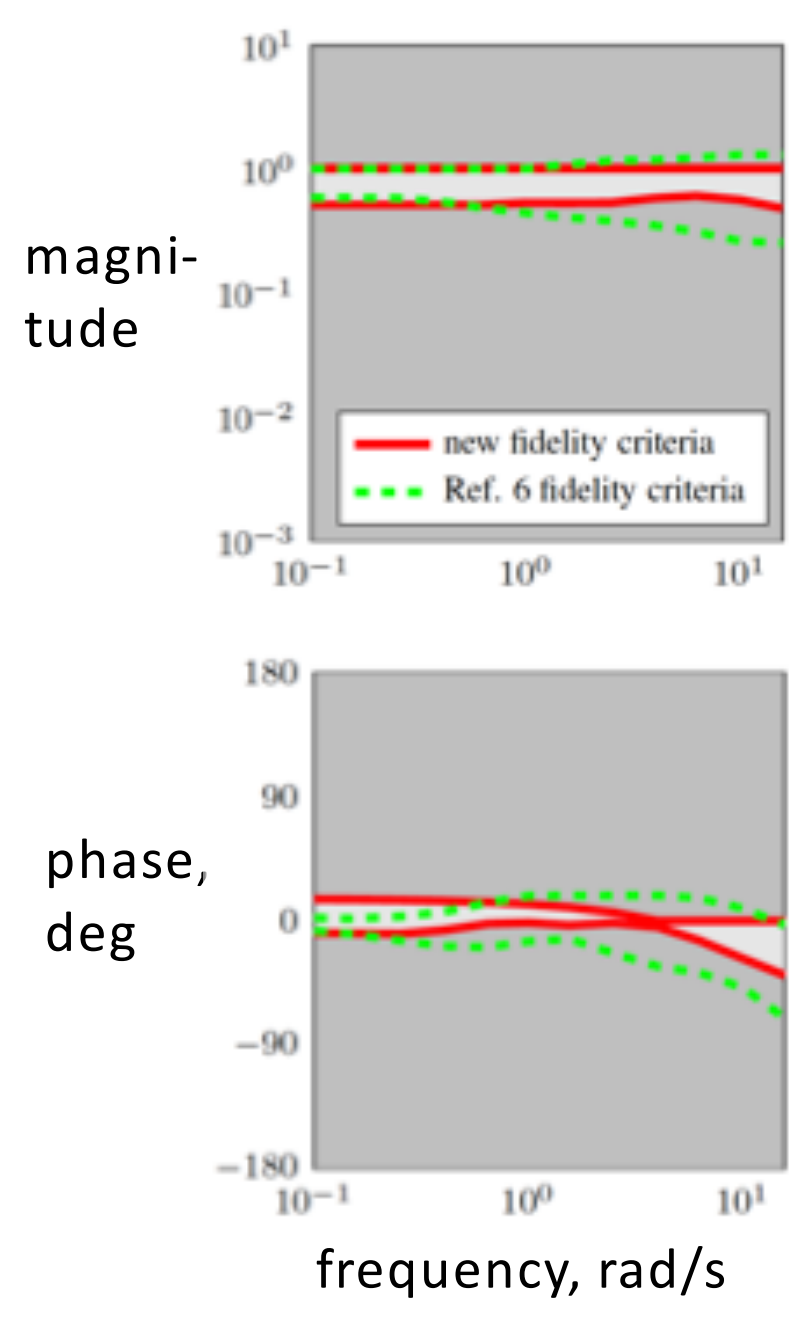

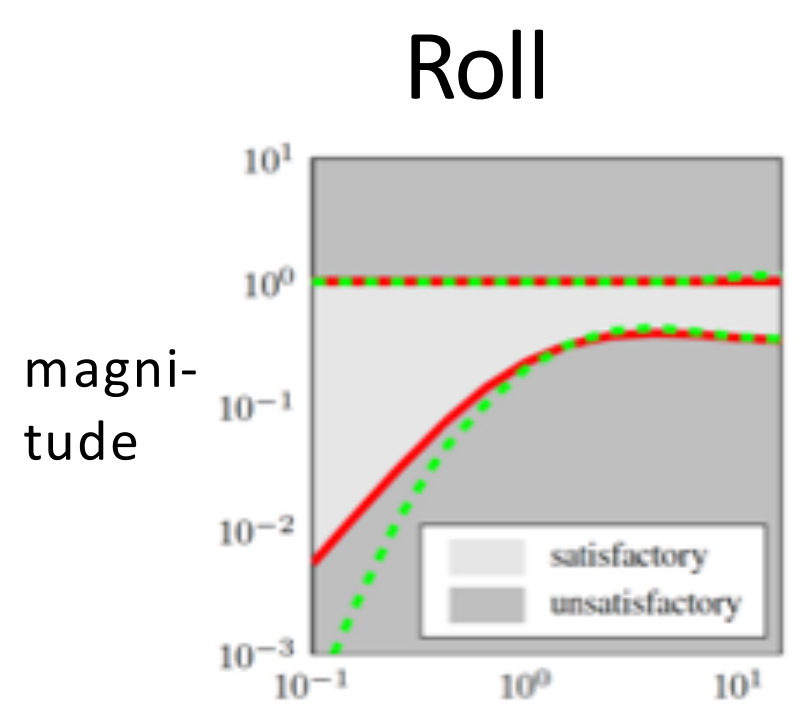
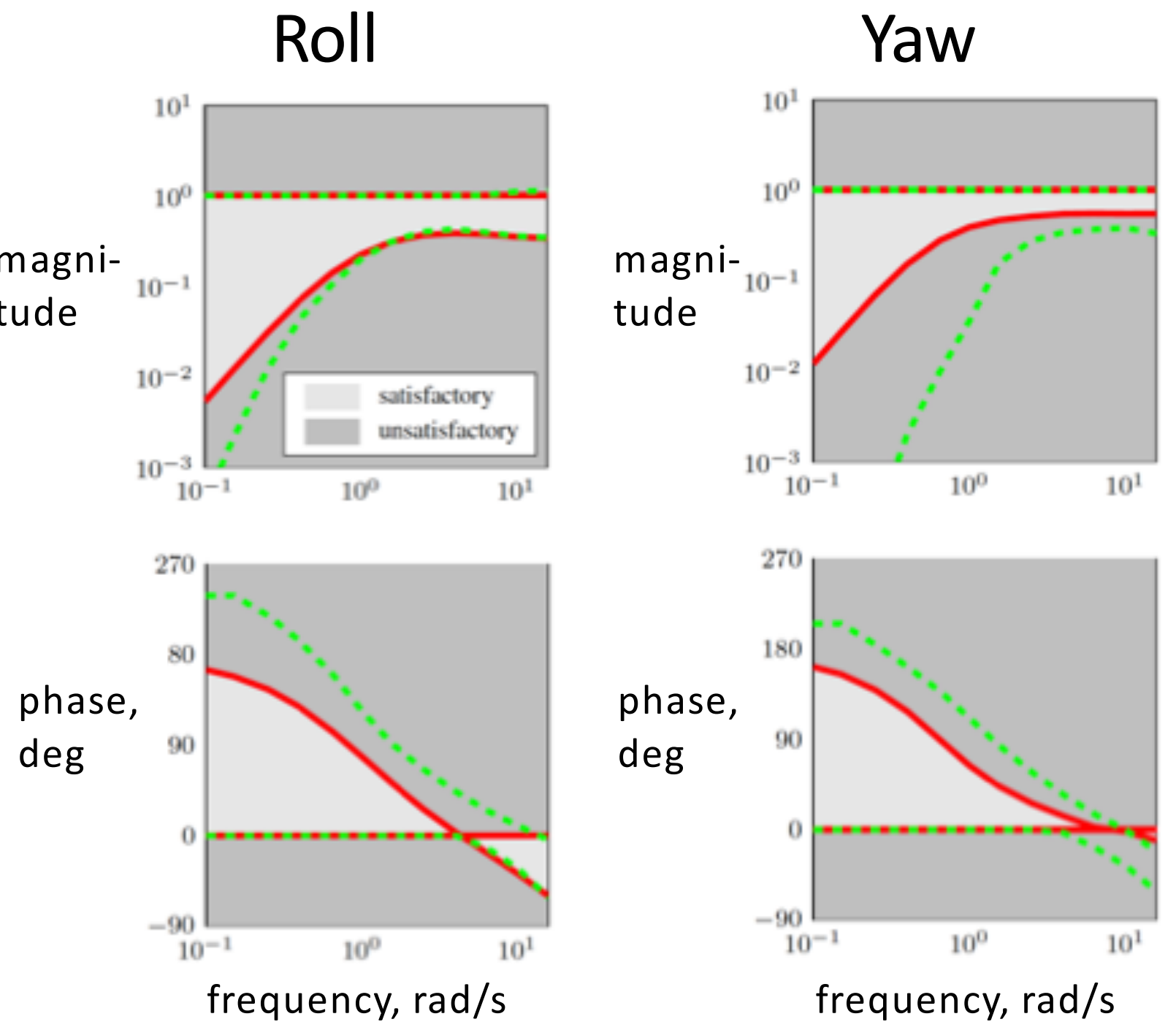


\section{Translational Motion Cueing Bounds}

Surge

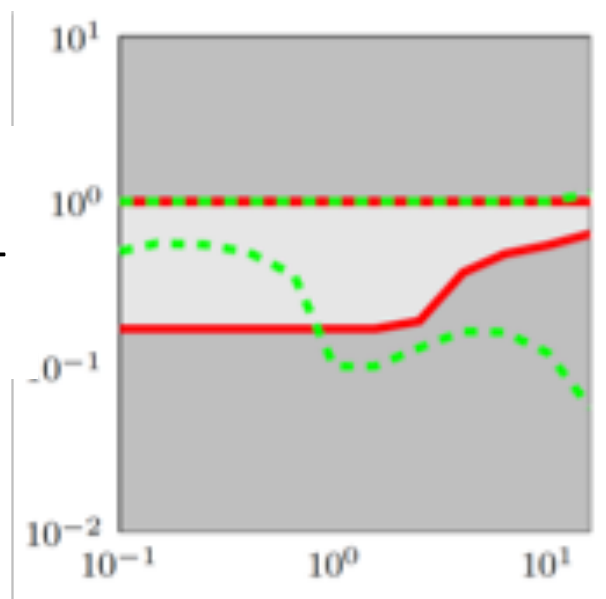

phase, deg

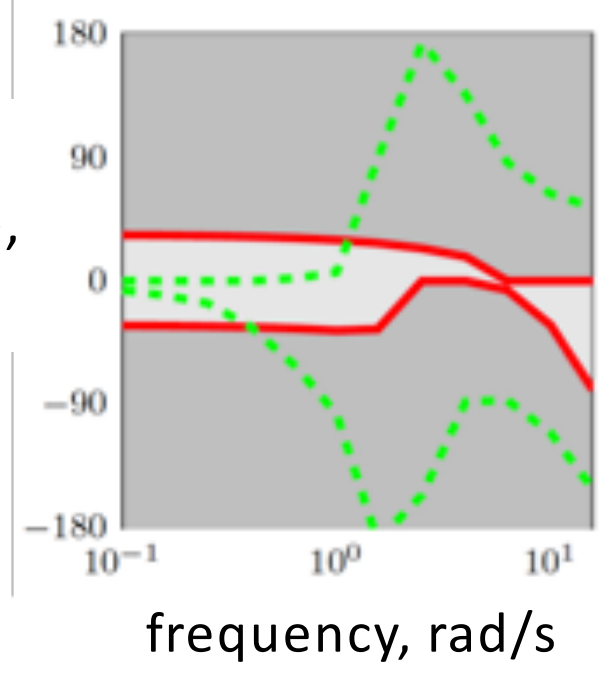

Sway
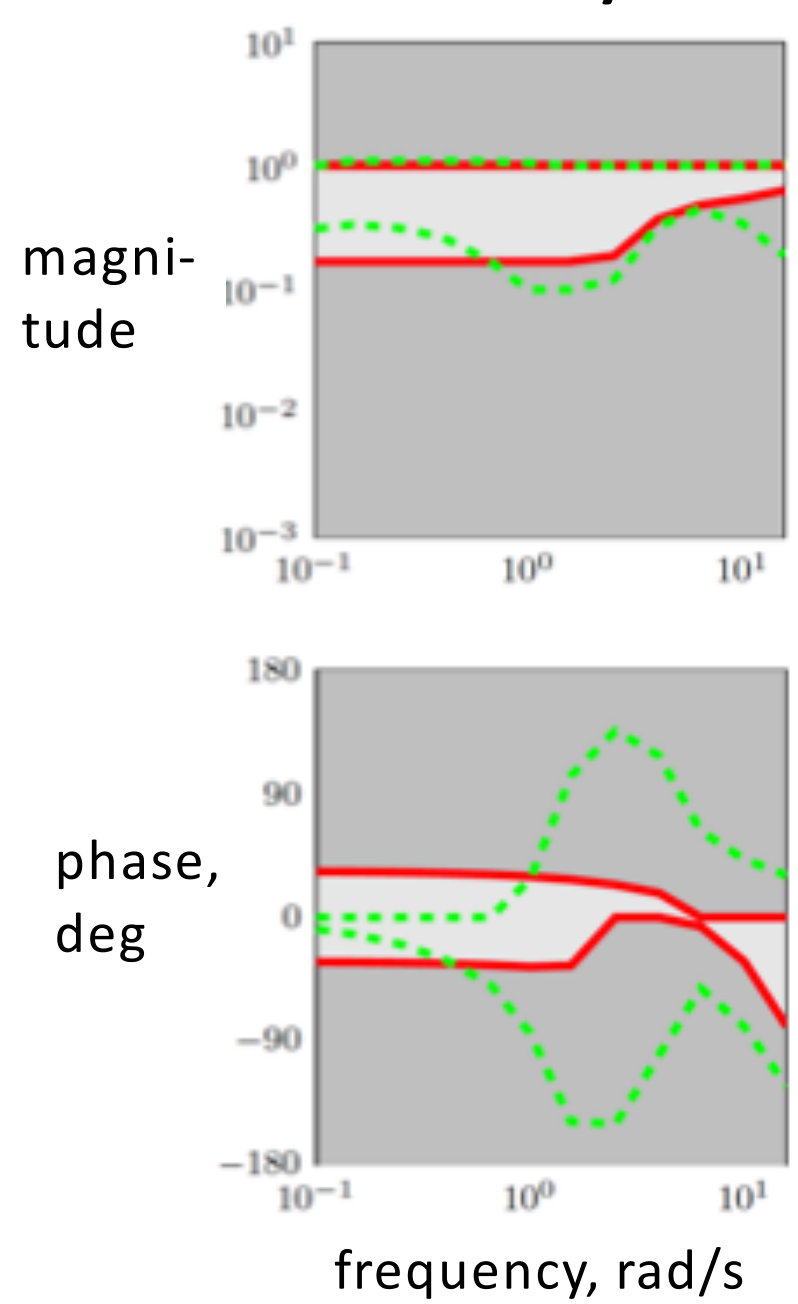

Heave

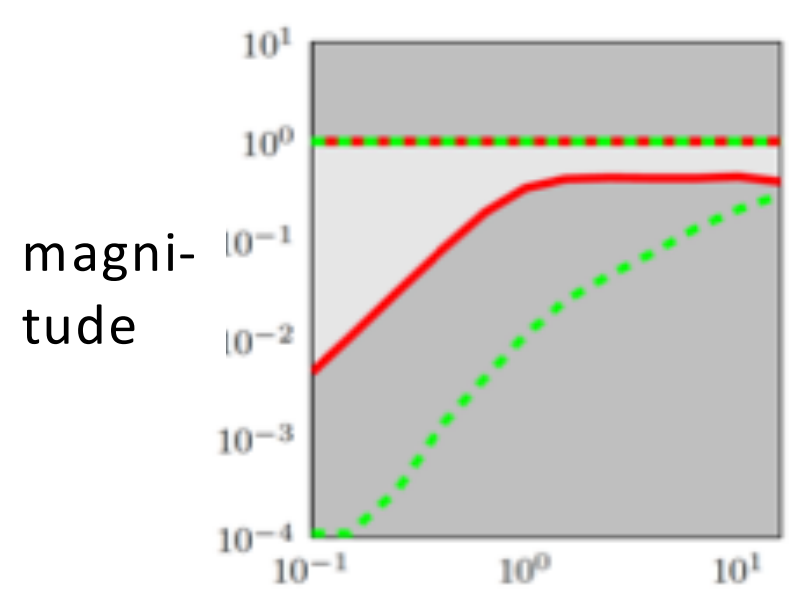

phase, deg

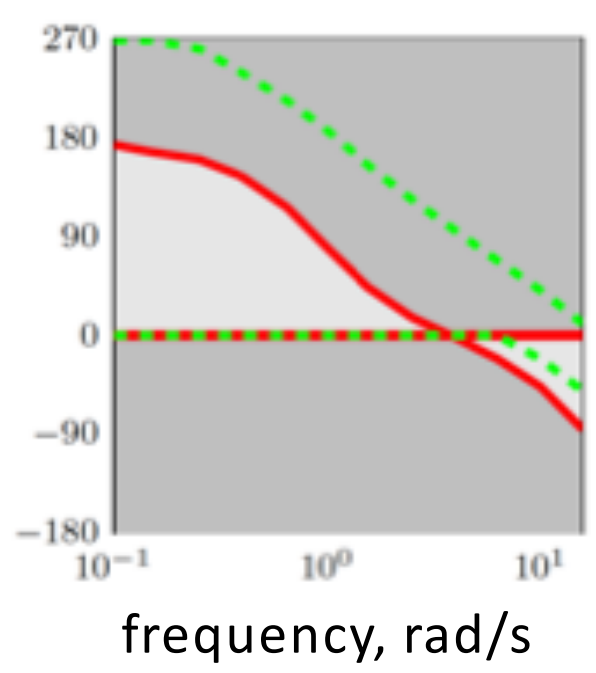




\section{Cross-Coupling Bounds}

\section{Roll to sway}
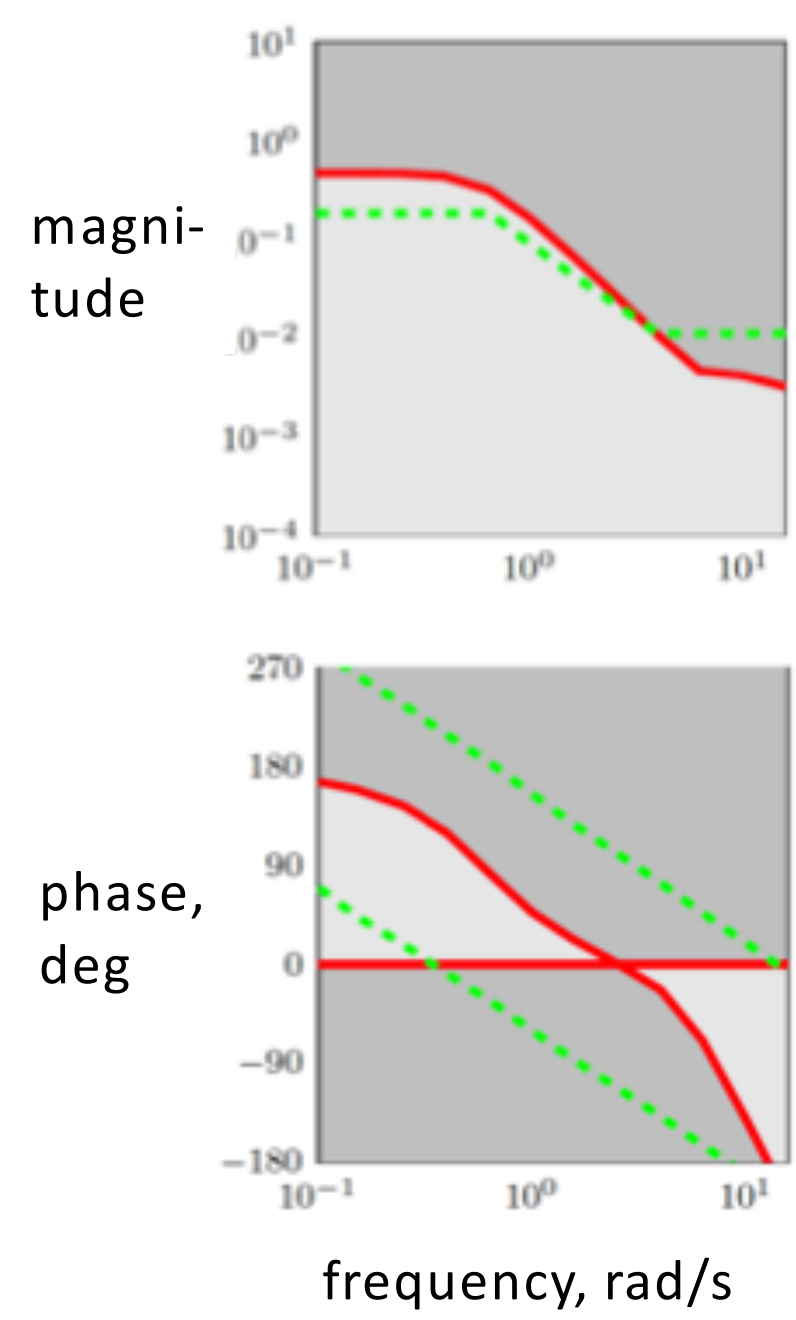

\section{Surge to pitch}
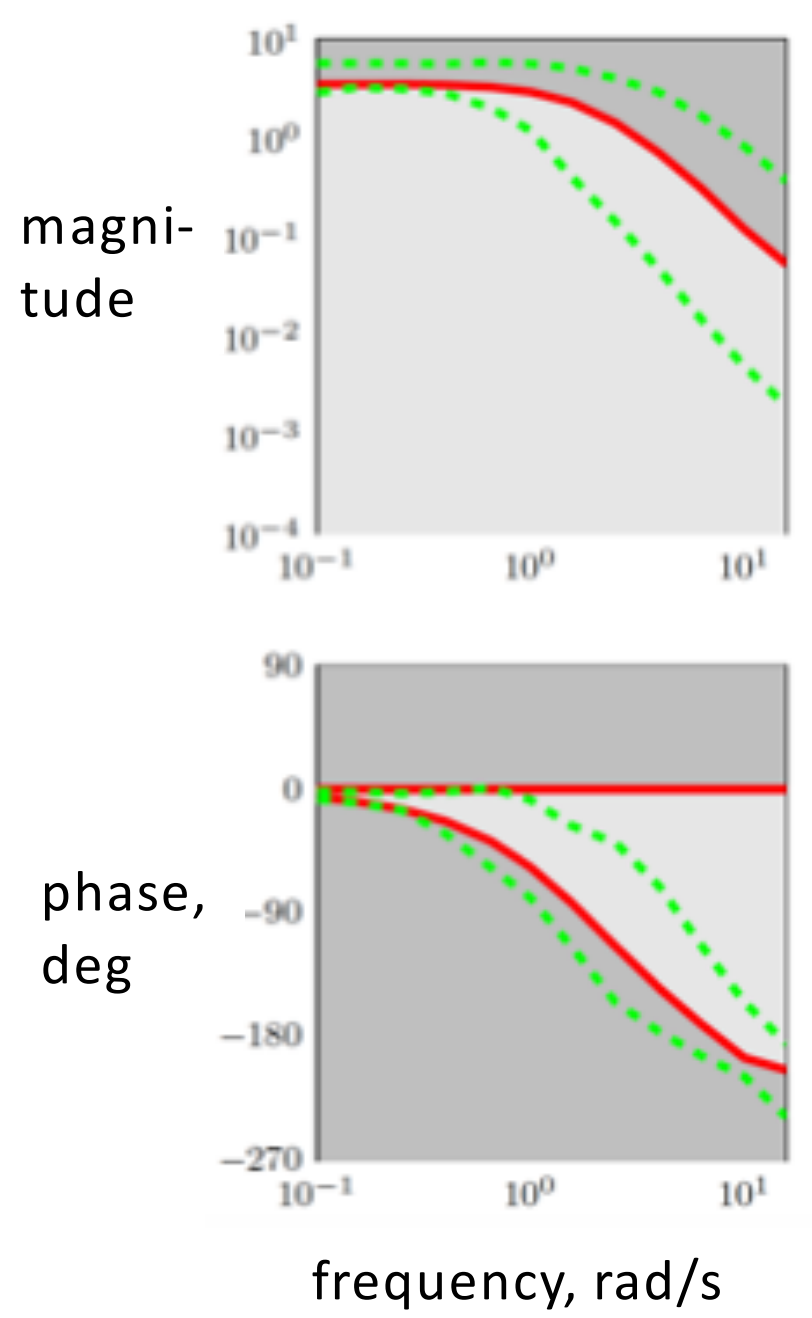


\section{Conclusions}

1. Motion condition significantly affected:

- Sinkrate at touchdown in the landing

- Roll deviation in the stall approach

- Maximum pitch rate in the stall recovery

- Heading deviation after the engine failure

- Pedal reaction time after the engine failure

2. False motion cues above the perceptual threshold resulted in higher false motion cue ratings in all tasks 


\section{Conclusions}

3. Significant differences between motion configurations defined initial objective motion cueing criteria

4. Initial comparison against data from one simulator manufacturer shows promise. Translational motion fidelity would need improvement 


\section{Objective Motion Cueing Criteria for Commercial Transport Simulators}

\section{AIAA-2018-2935}
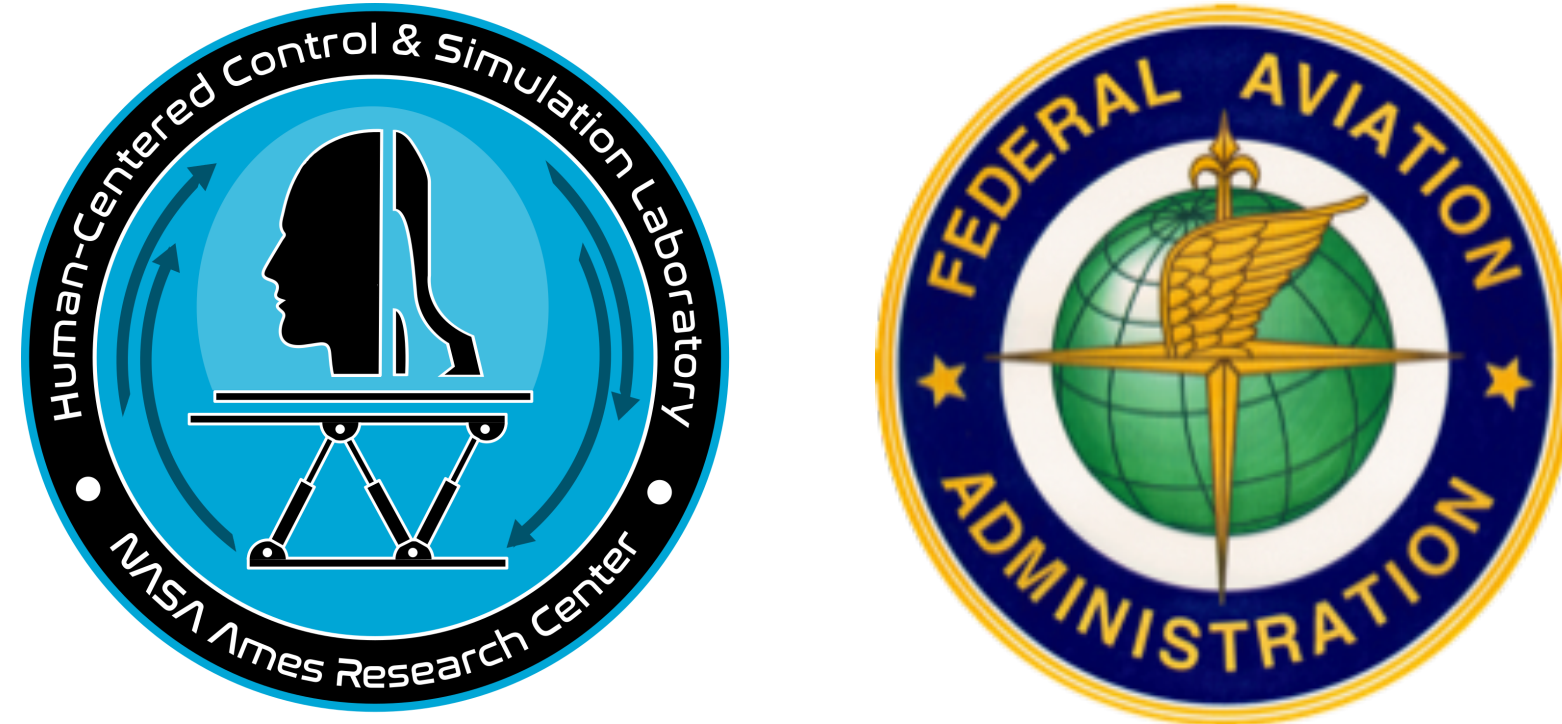

\section{Peter M. T. Zaal}

San José State University NASA Ames Research Center
Jeffery A. Schroeder

Federal Aviation Administration
William W. Chung Science Applications Int. Corp. NASA Ames Research Center 